Instrumented Taylor anvil-on-rod impact tests for validating applicability of standard strength models to transient deformation states

D. Eakins and N.N. Thadhani

Reprinted with permission from D. E. Eakins, Journal of Applied Physics, 100, 073503 (2006). Copyright 2006, American Institute of Physics. This article may be downloaded for personal use only. Any other use requires prior permission of the author and the American Institute of Physics. 


\title{
Instrumented Taylor anvil-on-rod impact tests for validating applicability of standard strength models to transient deformation states
}

\author{
D. E. Eakins and N. N. Thadhani ${ }^{\text {a) }}$ \\ School of Materials Science and Engineering, Georgia Institute of Technology, 771 Ferst Drive, \\ Love Building, Atlanta, Georgia 30332
}

(Received 12 December 2005; accepted 20 July 2006; published online 3 October 2006)

\begin{abstract}
Instrumented Taylor anvil-on-rod impact tests have been conducted on oxygen-free electronic copper to validate the accuracy of current strength models for predicting transient states during dynamic deformation events. The experiments coupled the use of high-speed digital photography to record the transient deformation states and laser interferometry to monitor the sample back (free surface) velocity as a measure of the elastic/plastic wave propagation through the sample length. Numerical continuum dynamics simulations of the impact and plastic wave propagation employing the Johnson-Cook [Proceedings of the Seventh International Symposium on Ballistics, 1983, The Netherlands (Am. Def. Prep. Assoc. (ADPA)), pp. 541-547], Zerilli-Armstrong [J. Appl. Phys. C1, 1816 (1987)], and Steinberg-Guinan [J. Appl. Phys. 51, 1498 (1980)] constitutive equations were used to generate transient deformation profiles and the free surface velocity traces. While these simulations showed good correlation with the measured free surface velocity traces and the final deformed sample shape, varying degrees of deviations were observed between the photographed and calculated specimen profiles at intermediate deformation states. The results illustrate the usefulness of the instrumented Taylor anvil-on-rod impact technique for validating constitutive equations that can describe the path-dependent deformation response and can therefore predict the transient and final deformation states. () 2006 American Institute of Physics. [DOI: 10.1063/1.2354326]
\end{abstract}

\section{INTRODUCTION}

The response of a material to high-strain-rate loading is an important subject, owing to the innumerable applications for materials in dynamic environments, such as high-rate forming, ballistics, and impact on vehicle structures. Understanding this behavior is essential for predictive purposes, reducing the iterative nature of designing components. The heart of predictive material modeling is the constitutive equation, which relates the application of load to deformation response on the basis of material properties. The validity of the constitutive equation depends upon its ability to describe fully and predictively any experimentally obtained information throughout the deformation event (and not just the end state) as a function of strain, strain rate, and temperature. Several of the more widely used constitutive equations have either empirical forms resulting from test data spanning a certain range of strain rates, ${ }^{1}$ or are based on physically based models relying on effects of thermally activated dislocation motion. ${ }^{2-4}$

The rod-on-rigid-anvil impact experiment performed by Taylor in 1948 remains a popular method of investigating the mechanical response of a material to dynamic loading. ${ }^{5}$ In the original configuration, a cylindrically shaped specimen is impacted against a rigid anvil at velocities sufficient to induce plastic deformation without failure, where strain rates in the range of $10^{3}-10^{5} \mathrm{~s}^{-1}$ are typical. By comparing the shape (length change) between the final deformed and initial specimens for a given impact velocity, estimates of an average dynamic flow stress of the material may be made. Early

${ }^{a)}$ Electronic mail: naresh.thadhani@mse.gatech.edu work on the construction/validation of constitutive relationships was similarly based upon comparisons between numerically simulated and experimentally obtained deformed specimen geometry. ${ }^{1,2,6-8}$ However, such comparisons do not provide a true validation, since the models are not used to generate the deformation path (just the end state) and the estimate of dynamic yield strength is based on the assumption of perfectly plastic material response. The inability to record the evolving profile during the deformation event prevented a robust validation of the early constitutive models.

The modern Taylor test has evolved considerably from its early start. In recent years, use of high-speed digital photography has made the capture of the deformation history possible. With interframe times in some cases of as few as several tens of nanoseconds, the time-resolved transient specimen profiles permit the validation of material models that can describe deformation occurring over a range of time and not just limited to the final deformed state. In addition, experiments conducted in the reverse configuration, i.e., impacting a rigid anvil against a stationary rod, permit spatially dependent measurements to be made that would be impossible if the sample were otherwise in motion. For example, VISAR (velocity interferometry system for any reflector, Ref. 9) can be employed to monitor the particle velocity at the sample's back (free) surface or at nearly any location within the specimen periphery. ${ }^{10,11}$ Coupling the multiple forms of time-resolved measurement techniques, complementary information can be obtained against which current and future constitutive models can be more robustly evaluated.

To date, a number of popular strength models have been 


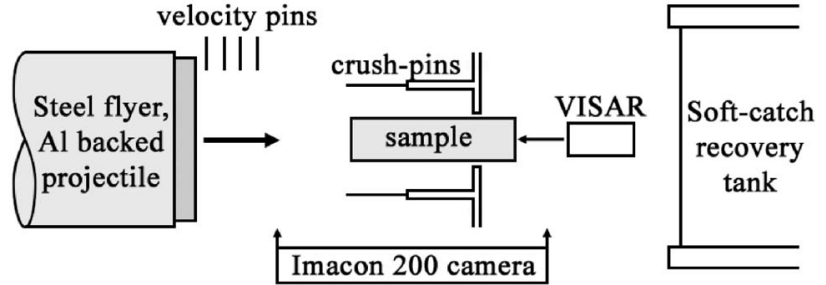

FIG. 1. Schematic of the instrumented Taylor anvil-on-rod impact experiment showing the arrangement of the projectile, sample, VISAR probe, and camera.

used to predict the final geometry of Taylor impact specimens tested over a range of strain rates. The models by Johnson and Cook, ${ }^{1}$ Zerilli and Armstrong, ${ }^{2}$ and Steinberg et $a l .{ }^{4}$ are some of the most widely known and frequently used, partly owing to their incorporation into many private and commercial hydrocodes. The applicability of these models to transient deformation states however, has been largely ignored. To address path-dependent effects, a few models have recently been developed, such as the modified ArmstrongZerilli model by Goldthorpe et $a .^{12}$ and Gould and Goldthorpe $^{13}$ and the empirical model of Frechard et al. ${ }^{14}$ Validation of these path-linked models through comparison with transient profiles has shown improvements over the traditional models. ${ }^{14,15}$ However, the use of approaches that provide complementary measures of transient deformation states and require adherence to transient phenomenon has yet to be adopted as a general rule for model validation.

The goal of the work described in this paper is to establish a method for validating constitutive equations using multiple time-resolved measurement techniques. Instrumented Taylor anvil-on-rod impact experiments were thus performed on an oxygen-free electronic (OFE) $\mathrm{Cu}$ standard at $83 \mathrm{~m} / \mathrm{s}$, utilizing high-speed digital photography to obtain transient deformation profiles and velocity interferometry to record the sample back surface velocity revealing the various elastic, plastic, and radial wave interactions. Continuum finite-element simulations of the deformation event were made using the Johnson-Cook empirical equation and the physically based Zerilli-Armstrong and Steinberg-Guinan models, and the constant parameters were adjusted in each case to match the experimentally derived VISAR trace. The three models with respective fitted constants were then compared with the final deformation state of the recovered sample and the recorded transient deformation profiles for the $83 \mathrm{~m} / \mathrm{s}$ experiment for further evaluation and validation. The models with the same constants were also applied to the results from the experiment conducted at $205 \mathrm{~m} / \mathrm{s}$ for evaluation and validation at the higher velocity.

\section{EXPERIMENTAL PROCEDURE}

\section{A. Instrumented Taylor anvil-on-rod impact test}

An $80 \mathrm{~mm}$ diameter $7.6 \mathrm{~m}$ long helium driven singlestage gas gun was used to perform the instrumented Taylor anvil-on-rod impact experiments (Fig. 1). The projectile is comprised of a $76.2 \mathrm{~mm}$ diameter rigid flyer plate, machined from $6 \mathrm{~mm}$ thick hardened maraging 300 steel, mechanically fastened to a $178 \mathrm{~mm}$ long solid aluminum carrier sabot. All contacting surfaces were lapped prior to assembly. A wraparound breach firing mechanism was used to propel the projectiles at velocities of 83 and $205 \mathrm{~m} / \mathrm{s}$, measured by a series of shorting pins located at the muzzle face. To avoid unwanted specimen damage imparted by secondary impacts, a soft-catch recovery technique was used in order to slow the projectile shortly after impacting the sample. An Imacon 200 high-speed digital framing camera capable of $50 \mathrm{~ns}$ interframe time was positioned at one of the experiment chamber windows to record the deformation event, while a flash placed at the opposite side was used to achieve backlighting. The free surface velocity interferometry measurements were collected by a VISAR probe positioned approximately $30 \mathrm{~mm}$ from the specimen back surface and recorded on a gigahertz frequency oscilloscope. Triggering of the camera and the digital oscilloscope for the VISAR was accomplished using Dynasen end-impact crush pins placed $2 \mathrm{~mm}$ preceding the specimen impact face.

\section{B. Specimen preparation}

OFE grade copper (C10100) was supplied as $\frac{3}{4}$ in. diameter round rod as specified by ASTM B187. Cylindrical Taylor specimens were prepared from the stock observing a length to diameter ratio of $4: 1$ chosen for the series of experiments. The ends of the rods were similarly lapped parallel to within $0.008^{\circ}$ using $15 \mu \mathrm{m}$ diamond suspension for a rough polish. The remaining cylinder surface was untreated and had a smooth unpolished mill finish. The copper rods were supported by a 1/8 in. thick poly methyl methacrylate (PMMA) disk, offset from the specimen back surface by $9 \mathrm{~mm}$ to allow imaging of the sample's entire length. Perpendicularity between the sample face and barrel axis to within $3 \mathrm{mrad}$ was achieved for each experiment through the use of a two-axis target adjustment ring and laser alignment system.

\section{Experimental measurements}

The high-speed framing camera allows the transient profiles to be captured at any time during the deformation event. Use of backlighting has become a standard in increasing the contrast between the specimen and surrounding environment, reducing the uncertainty in measurements. A total of 16 frames were captured in each experiment, each image consisting of $1200 \times 980$ pixels. Most frames were spent at the early stages of deformation while the free end was still in view. The first two frames captured the sample and projectile before impact, providing another measure of the impact velocity. The next 11 frames were spaced evenly at short time intervals while maintaining full visibility of the back surface. Images captured with the back end obscured or beyond the framing window were undesirable, since the specimen length could not be determined for data analysis. The remaining three frames were spent at later times for the purpose of determining the time of separation between projectile and sample.

Using VISAR, the free surface velocity of a specimen may be recorded during impact, giving an accurate measurement of particle velocity $U_{p}$ and overall back-end speed $b$. 


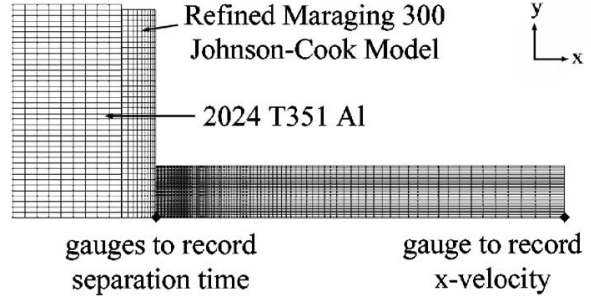

FIG. 2. AUTODYN-2D meshes used for all simulations with graded mesh density toward the impact surface and gauge points for recording $x$ velocity at the impact and free surface.

Unlike the camera images, which are limited in number and resolution, VISAR data evolve throughout the deformation event and are dependent on the mechanical wave behavior and their interactions during propagation across the sample length. For the experiments using VISAR, a green laser interferometry system manufactured by VALYN and nanosecond resolution recording devices were used.

\section{AUTODYN-2D simulations}

Following each experiment, dynamic deformation simulations using the experimental impact velocity were run with the AUTODYN-2D hydrocode originally developed by Century Dynamics. ${ }^{16}$ AUTODYN-2D is a commercial finite-element package that specializes in the computation of dynamic simulations and contains a variety of solvers [Lagrange, Euler, arbitrary lagrangian eulerian (ALE), and smoothed particle hydrodynamics (SPH)]. Of those available, the Lagrangian solver is most appropriate for simulating deformations where the strains are small. An axisymmetric model of the reverse-Taylor experiment was constructed utilizing a lower- $I$ graded mesh for the copper specimen (Fig. $2)$. A mesh density study indicated that the resolution employed $(0.2 \mathrm{~mm}$ at the impact face) was adequate to converge upon steady elastic and plastic responses. To simulate the VISAR data recorded from the back surface, a gauge was placed on the last node to record the velocity component in the $x$ direction. Additional gauges were also placed in adjacent nodes of the flyer and specimen impact end to assist in determining the time of separation between projectile and sample. As a first approximation, the Johnson-Cook and Zerilli-Armstrong viscoplastic models [Eqs. (1) and (2)] and the Steinberg-Guinan strength model popularly used for the high-strain-rate regime [Eqs. (3) and (4)] for OFHC copper were used to simulate the deformation response of $\mathrm{OFE} \mathrm{Cu}$ samples investigated in this study:

$$
\begin{aligned}
\sigma= & \left(\sigma_{0}+B \varepsilon^{n}\right)\left(1+C \ln \dot{\varepsilon}^{*}\right)\left(1-T^{* m}\right), \\
\sigma= & \sigma_{G}+C_{2} \varepsilon^{1 / 2} \exp \left(-C_{3} T+C_{4} T \ln \dot{\varepsilon}\right)+k d^{-1 / 2}, \\
\sigma= & \sigma_{0}\left[1+\beta\left(\varepsilon+\varepsilon_{i}\right)\right]^{n} \\
& \times\left[1+\left(\frac{\sigma_{P}^{\prime}}{\sigma_{0}}\right) \frac{P}{\eta^{1 / 3}}+\left(\frac{G_{T}^{\prime}}{G_{0}}\right)(T-300)\right],
\end{aligned}
$$

TABLE I. Initial and final dimensions of OFE copper.

\begin{tabular}{ccccc}
\hline \hline $\begin{array}{c}\text { Velocity } \\
(\mathrm{m} / \mathrm{s})\end{array}$ & $\begin{array}{c}\text { Initial } \\
\text { diameter, } d_{0} \\
(\mathrm{~mm})\end{array}$ & $\begin{array}{c}\text { Initial } \\
\text { length, } l_{0} \\
(\mathrm{~mm})\end{array}$ & $\begin{array}{c}\text { Final } \\
\text { diameter, } d_{f} \\
(\mathrm{~mm})\end{array}$ & $\begin{array}{c}\text { Final } \\
\text { length, } l_{f} \\
(\mathrm{~mm})\end{array}$ \\
\hline 205 & 18.9 & 75.0 & 36.6 & 54.5 \\
83 & 19.1 & 75.1 & 23.6 & 70.3 \\
\hline \hline
\end{tabular}

$$
G=G_{0}\left[1+\left(\frac{G_{P}^{\prime}}{G_{0}}\right) \frac{P}{\eta^{1 / 3}}+\left(\frac{G_{T}^{\prime}}{G_{0}}\right)(T-300)\right] .
$$

The parameters in the above equations correspond to standard terminology. $1,2,4$

Adjustments to the starting model parameters were made until the simulated velocity trace matched the experimental VISAR data. Simulated images of the specimen profiles were then constructed for the final deformed state, and at times coinciding with the experimental camera data, to determine if the model followed the same deformation path.

An adjusted Johnson-Cook strength model incorporating several measured elastic constants was used for the steel flyer and left unchanged for each simulation. Though the validity of the Johnson-Cook model is under scrutiny, the choice of strength model for the maraging steel flyer plate bears little weight since it is assumed to be nearly rigid.

\section{RESULTS AND DISCUSSION}

Impact experiments on OFE copper were performed at 83 and $205 \mathrm{~m} / \mathrm{s}$ to showcase the extremes in deformation response (ever-convex profile at $83 \mathrm{~m} / \mathrm{s}$, concave/convex transition and barreling at $205 \mathrm{~m} / \mathrm{s}$ ). The lengths and diameters of the initial and final impacted rods from each experiment are listed in Table I, where the final diameter refers to that of the crushed end.

\section{A. Experimental camera and free surface velocity data}

Selected high-speed camera images showing the transient deformation states for the experiment performed at an impact velocity of $83 \mathrm{~m} / \mathrm{s}$ are presented in Fig. 3. For the field of view shown, each pixel corresponds to approximately $81 \mu \mathrm{m}$ for an image resolution of $12.3 \mathrm{pixel} / \mathrm{mm}$. The images reveal deformation occurring without movement of the free surface until perhaps $58 \mu \mathrm{s}$; however, determining the exact time of movement from camera data is complicated by the variable perspectives of each image channel. Separation between the sample and flyer appears to occur between 134.7 and $169.2 \mu \mathrm{s}$, as shown from the later images in the inset of Fig. 3.

The free surface velocity trace recorded by VISAR from the $83 \mathrm{~m} / \mathrm{s}$ experiment is shown in Fig. 4, where the onset of disturbance at the free surface is chosen for the start time, $t_{0}$. The free surface reaches a velocity of $80 \mathrm{~m} / \mathrm{s}$ nearly $100 \mu \mathrm{s}$ after $t_{0}$ before the signal is lost. The oscillations early in the trace are caused by the alternating states of tension and compression at the back surface due to radial reflection of the elastic waves, and are most pronounced when the VISAR probe is focused at the center of the rod's back face. 

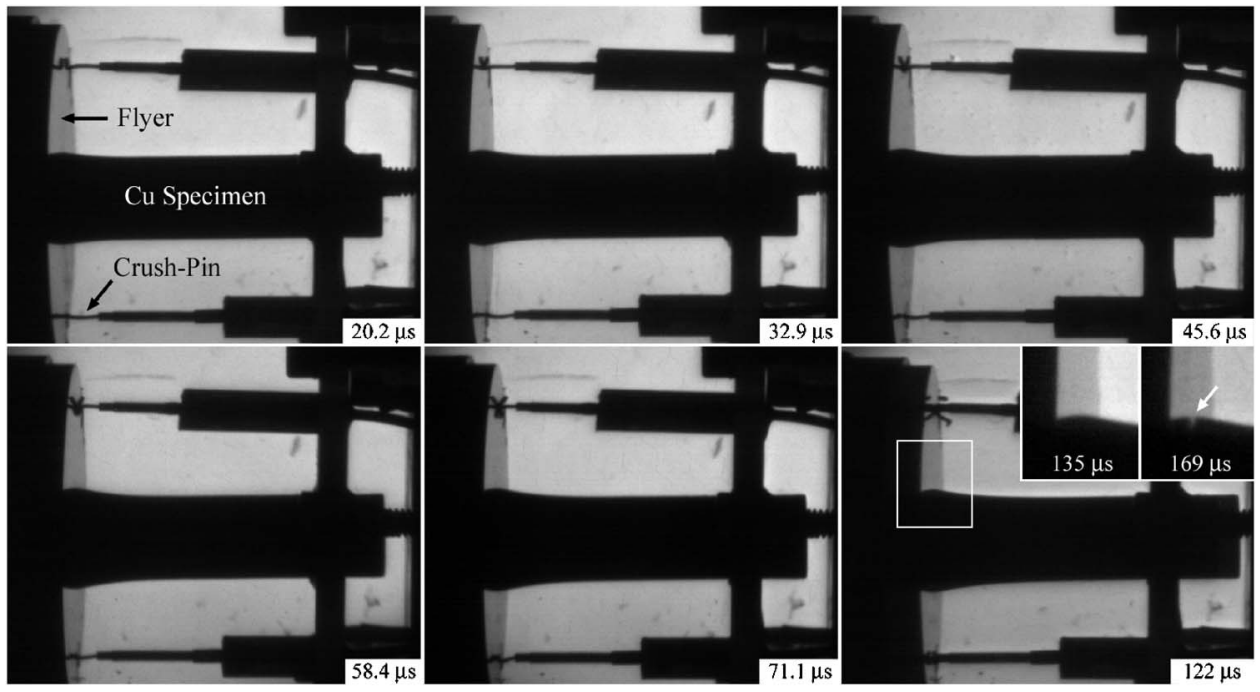

FIG. 3. High-speed digital images capturing the transient deformation profiles of the $83 \mathrm{~m} / \mathrm{s}$ experiment, where times shown are referenced from impact. Separation between the flyer and specimen occurs between 135 and $169 \mu$ s, as shown in the inset.

\section{B. Simulations and model refinement}

Simulations using the AUTODYN-2D hydrocode with Johnson-Cook (JC), Zerilli-Armstrong (ZA), and SteinbergGuinan (SG) models were first used to fit the experimentally recorded VISAR velocity trace recorded for the $83 \mathrm{~m} / \mathrm{s}$ experiment. Using the fitted parameters, the simulations were used to obtain the final deformed state and transient deformation profiles of the $83 \mathrm{~m} / \mathrm{s}$ impacted sample.

\section{Free surface velocity simulations}

Equivalent velocity records simulated using the JC, ZA, and SG models with parameters for oxygen-free high conductivity (OFHC) copper (available from the AUTODYN library) are shown as dashed lines along with the experimental data in Figs. 4(a)-4(c), for the experiment performed at $83 \mathrm{~m} / \mathrm{s}$. Each of the models produced characteristics similar to the experiment, exhibiting equivalently spaced oscillations early in the velocity traces. However, predicted rise times to $80 \mathrm{~m} / \mathrm{s}$ were considerably longer than the $100 \mu$ s rise time observed experimentally, indicating a lower degree of strength and strain hardening. These differences are not unexpected, since the parameters used in the models pertain to OFHC $\mathrm{Cu}$ and are not intended for the OFE $\mathrm{Cu}$ material tested in this work. Maintaining the form of the JohnsonCook, Zerilli-Armstrong, and Steinberg-Guinan equations, adjustments were made to the starting parameters until an agreeable match between the simulation and experiment was met. The resulting fitted VISAR traces following the changed constant parameters (listed in Table II along with original constants) are also shown as solid traces in Figs. 4(a)-4(c).

In the Johnson-Cook equation, the value of the workhardening exponent $n(=0.05)$ was determined from quasistatic compression tests of the starting material. Such a low value of the exponent is typical of cold-worked $\mathrm{Cu}^{17} \mathrm{De}$ creasing the hardening exponent had the effect of increasing the VISAR trace slope, in addition to increasing the amount of curvature. To compensate for the increase in curvature, changes in the hardening constant $C$ were made until good agreement was reached between the traces.
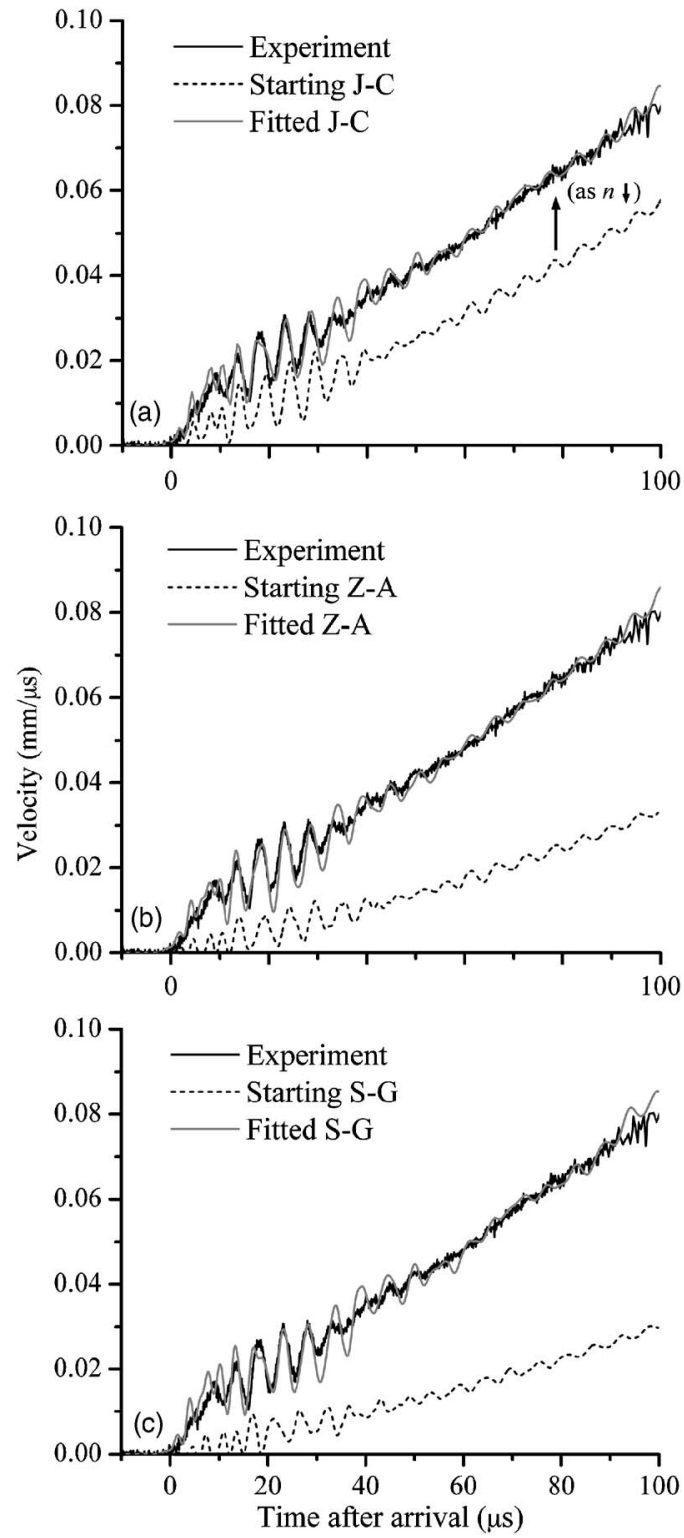

FIG. 4. Free surface velocity trace determined experimentally at $83 \mathrm{~m} / \mathrm{s}$ and through simulation utilizing the starting OFHC (dashed) and fitted (solid) (a) Johnson-Cook, (b) Zerilli-Armstrong, and (c) Steinberg-Guinan strength models. 
TABLE II. Constitutive model adjustments through VISAR matching.

\begin{tabular}{|c|c|c|c|c|c|c|c|}
\hline \multirow[b]{2}{*}{ Parameter } & \multicolumn{2}{|c|}{ Johnson-Cook } & \multicolumn{2}{|c|}{ Zerilli-Armstrong } & \multicolumn{3}{|c|}{ Steinberg-Guinan } \\
\hline & C & $n$ & $\sigma_{G}+k d^{1 / 2}(\mathrm{MPa})$ & $C_{3}$ & $\sigma(\mathrm{MPa})$ & $\beta$ & $n$ \\
\hline Starting & 0.025 & 0.31 & 65 & 0.0028 & 120 & 36 & 0.45 \\
\hline Fitted & 0.005 & 0.05 & 280 & 0.004 & 320 & 5 & 0.3 \\
\hline
\end{tabular}

Similarly, the yield strength determined through the same compression test was used to refine the Hall-Petch strength in the Zerilli-Armstrong model. Afterwards, the value of $C_{3}$ in the $\mathrm{ZA}$ equation was increased to reduce the curvature in the simulated trace. Fitting of the SteinbergGuinan model was more iterative. Though the best fit yielded strength parameters $Y$, hardening constant $\beta$, and hardening exponent $n$ that did not exactly match those determined by compression tests, the physical significance behind the modifications (increased yield strength, decreased hardening) was consistent.

In each case, fitting of the VISAR traces was achieved by reducing the number of free variables through experimentation, but it still remains an iterative process. The fits reached through this method are not necessarily exclusive and should not rule out the possibility of alternate parametric solutions to the experimental VISAR results.

\section{Transient and final deformation state simulations}

Before comparing the simulated profiles to the transient deformation states captured by the high-speed digital camera, an initial comparison of the final sample dimensions of the $83 \mathrm{~m} / \mathrm{s}$ experiment and those simulated using the three models was conducted. Table III lists the final crushed-end diameters, lengths, and associated errors using the original and fitted parameters for the Johnson-Cook, ZerilliArmstrong, and Steinberg-Guinan models. It can be seen that with the use of original parameters, each model exhibits good prediction of final impact face diameter $(0.6 \%-1.3 \%$ error), but varying degree of error in specimen length $(\sim 2.1 \%$ error for JC model, $\sim 5.9 \%$ error for ZA model, and $\sim 4.9 \%$ error for SG model).

Use of model parameters obtained following fitting of the free surface velocity traces to the VISAR data demonstrated substantial improvements in the predicted final length with less than a $1 \%$ difference with each model, while errors in impact-face diameter showed marginal increase $(\sim 3.2 \%$ for JC, $2.6 \%$ for ZA, and $0.87 \%$ for SG). These results suggest that the VISAR trace collected from the sample back (free) end is most sensitive to overall changes in length and may be used to improve the final fit of the current models. The final simulated specimen geometries are shown along with the recovered sample images in Fig. 5(a). The comparisons reveal a fit within the resolution limit of the digital images.

The transient specimen lengths for each selected frame shown in Fig. 3 for the $83 \mathrm{~m} / \mathrm{s}$ experiment, and their comparisons with simulated results using the fitted parameters are shown in Table IV. In each case, the match observed with fitted parameters for the respective models throughout the deformation event is well below $1 \%$. These results reemphasize that VISAR data collected from the free end capture the elastic and plastic disturbances responsible for length changes.

\section{Constitutive model validation of transient states}

Comparisons between the photographed and simulated crushed-end profiles (using fitted parameters with each of the models) at several stages during deformation in the case of the $83 \mathrm{~m} / \mathrm{s}$ sample are shown in Figs. 5(b)-5(f). The Johnson-Cook empirical model, with the fitted constants, follows the crushed-end diameter fairly well and overall shows a better match to the experimental profiles in comparison to the ZA and SG models. Though it appears that the model has still not fully converged upon complete agreement with the recorded data, the results are almost entirely within the uncertainty in camera measurements. While the Johnson-Cook model performs best at the impact face, the fitted SteinbergGuinan model does particularly well in matching the region immediately following the flared end. In contrast, the tran-

TABLE III. Final specimen dimensions and associated error for $83 \mathrm{~m} / \mathrm{s}$ experiment.

\begin{tabular}{|c|c|c|c|c|c|}
\hline & Expt. & Starting & Error $(\%)$ & Fitted & Error $(\%)$ \\
\hline \multicolumn{6}{|c|}{ Johnson-Cook } \\
\hline Diameter (mm) & 23.6 & 23.3 & 1.31 & 24.4 & 3.18 \\
\hline Length (mm) & 70.3 & 68.8 & 2.12 & 70.1 & 0.30 \\
\hline \multicolumn{6}{|c|}{ Zerilli-Amstrong } \\
\hline Diameter (mm) & 23.6 & 23.4 & 1.00 & 23.0 & 2.62 \\
\hline Length (mm) & 70.3 & 66.2 & 5.90 & 70.0 & 0.43 \\
\hline \multicolumn{6}{|c|}{ Steinberg-Guinan } \\
\hline Diameter (mm) & 23.6 & 23.776 & 0.60 & 23.428 & 0.87 \\
\hline Length (mm) & 70.3 & 66.84 & 4.93 & 70.059 & 0.35 \\
\hline
\end{tabular}


(a)
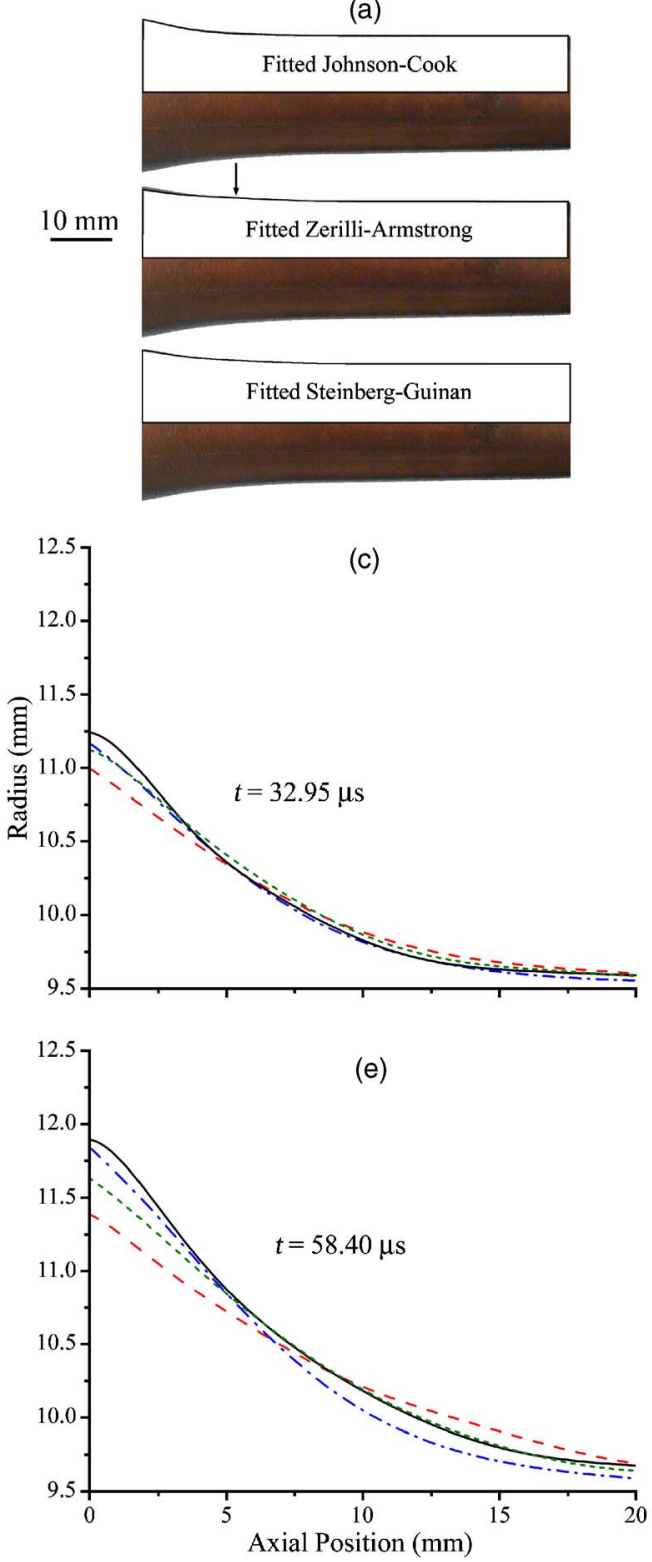

(b)

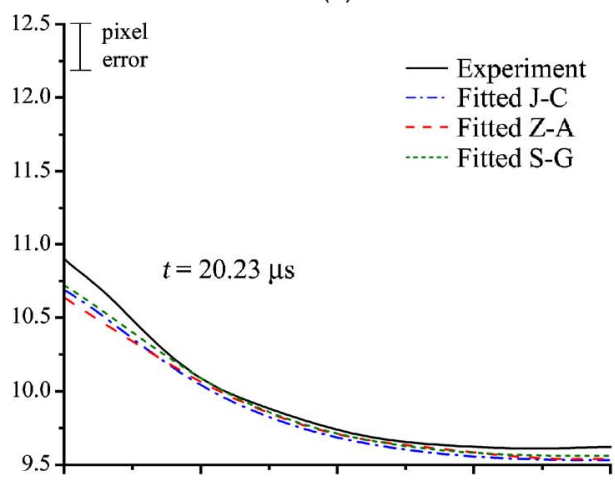

(d)

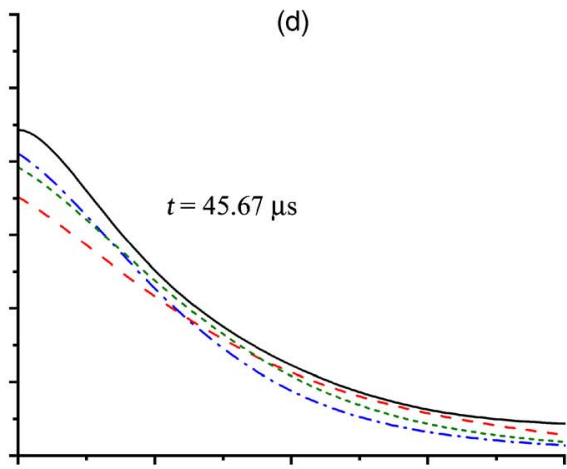

(f)

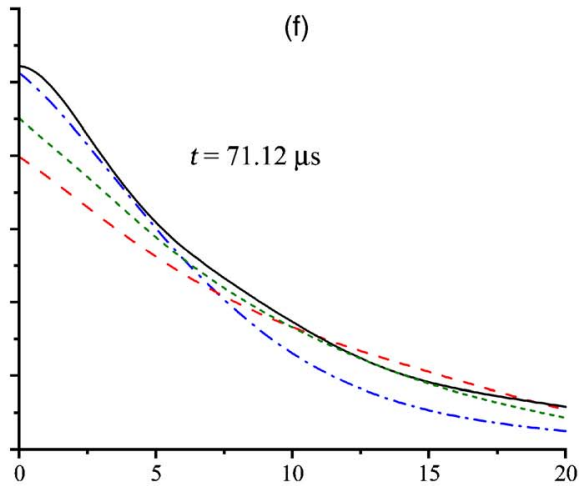

FIG. 5. (Color online) Comparison of the experimental and simulated profiles using the fitted Johnson-Cook, Zerilli-Armstrong, and Steinberg-Guinan strength models at the (a) final deformed state and [(b)-(f)] transient states. The fitted Johnson-Cook model matches the experimental profile almost entirely within the limits of measurement uncertainty (pixel error), while the fitted Steinberg-Guinan model closely follows the latter rod region, only slightly underestimating the crushed-end radius. The fitted Zerilli-Armstrong model underpredicts the crushed-end radius at all stages of deformation and exaggerates the deformation in the region immediately following the impact face [arrow in (a)].

sient deformation profiles obtained using the ZerilliArmstrong model with the fitted parameters reveal a much poorer agreement with the observed transient deformation profiles. The model greatly underpredicts the instantaneous crushed-end diameter and exaggerates the extent of deforma-

TABLE IV. Transient specimen lengths in $\mathrm{mm}$.

\begin{tabular}{ccccc}
\hline \hline Time $(\mu \mathrm{s})$ & Expt. & JC fit (\% error) & ZA fit $(\%$ error) & SG fit (\% error) \\
\hline 20.23 & 73.4 & $73.3(0.11)$ & $73.4(0.03)$ & $73.5(0.15)$ \\
32.95 & 72.5 & $72.5(0.03)$ & $72.5(0.02)$ & $72.7(0.26)$ \\
45.67 & 71.7 & $71.7(0.08)$ & $71.7(0.06)$ & $71.9(0.25)$ \\
58.40 & 71.1 & $71.0(0.08)$ & $71.1(0.01)$ & $71.3(0.33)$ \\
71.12 & 70.6 & $70.5(0.13)$ & $70.5(0.12)$ & $70.7(0.13)$ \\
\hline \hline
\end{tabular}

tion in the latter portion of the specimen. The results reveal that while constitutive equations validated against free surface velocity measurements can predict the final deformed state of impacted samples within a few percent error, the transient deformation states are not equally well predicted.

\section{Validation of transient and end states at high velocity}

While the fits to transient profiles were improved by matching the experimentally recorded free surface velocity traces (most notably in refining the length), it remains to be seen whether the performance of the fitted models can be extended to a range of velocities, or rather if it is unique to the fitting velocity $(83 \mathrm{~m} / \mathrm{s}$ in this case). To investigate this, 

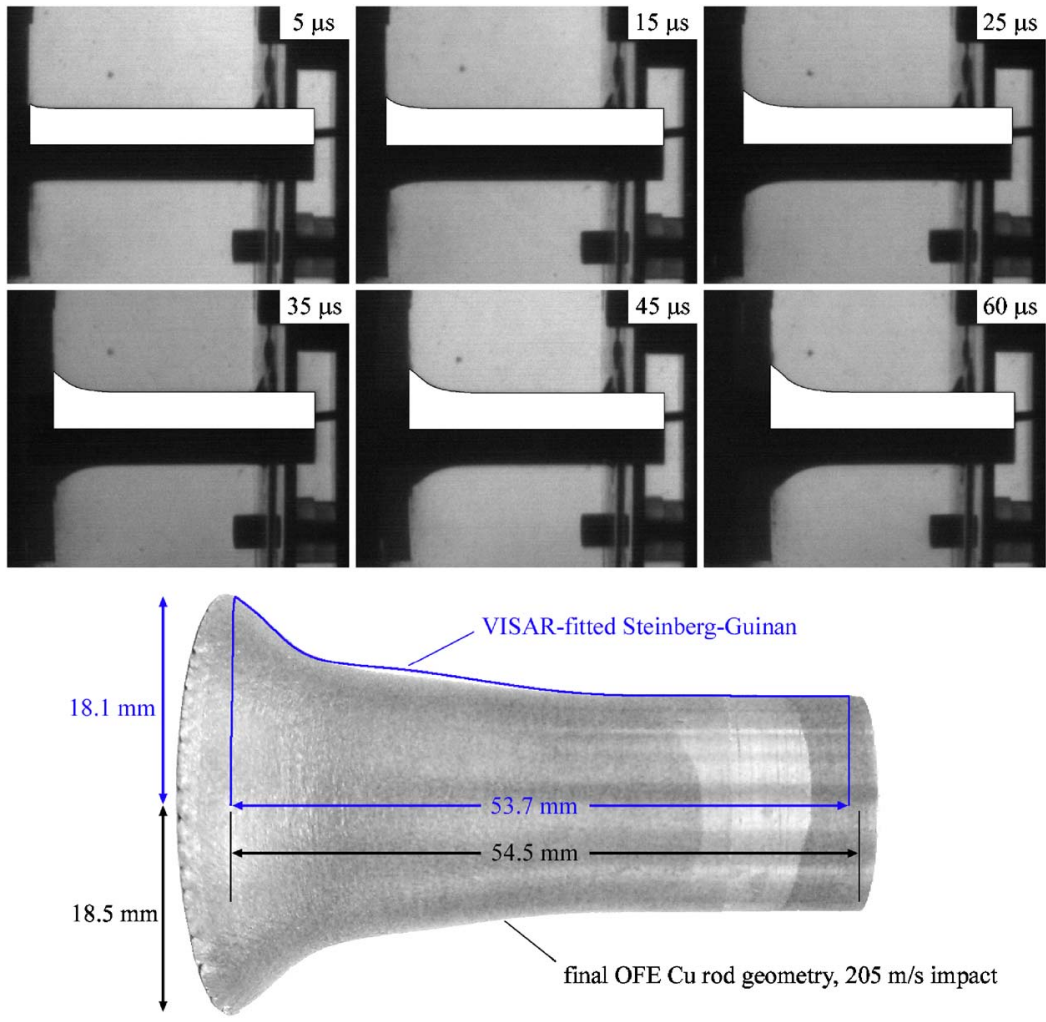

FIG. 6. (Color online) Transient and final deformed profiles of the $205 \mathrm{~m} / \mathrm{s}$ experiment simulated using the fitted Steinberg-Guinan model, as compared to highspeed camera data and recovered specimen. The simulated transient profiles (white) match the experimental images to within 5\% at early stages of deformation and does well to predict the barreled zone at the final state.

the VISAR fitted JC, ZA, and SG models for the $83 \mathrm{~m} / \mathrm{s}$ experiment were employed to simulate the transient and final profiles of the similar experiment performed at $205 \mathrm{~m} / \mathrm{s}$. The higher velocity produces a markedly different final profile, characterized by enhanced localized deformation in the region following the flared end (barreling).

The results of simulating the $205 \mathrm{~m} / \mathrm{s}$ experiment using the constants derived from the $83 \mathrm{~m} / \mathrm{s}$ experiment reveal that both the Zerilli-Armstrong and Steinberg-Guinan fitted models are able to reproduce the entire specimen profile (every location along the rod length) to within 5\% throughout the deformation event. Furthermore, the average error at any given frame is less than $2 \%$ for the fitted SG model and less than $3 \%$ for the ZA model. The fitted Johnson-Cook model, on the other hand, does not predict the formation of a barreled region, and suffers from considerably larger errors. Figure 6 shows a comparison of the transient frames at early times (up to $60 \mu$ s after impact) and the final deformed state based on the SG model, which shows the best match. Note the development of a barreled region closely, but not perfectly, matching that observed experimentally.

It should be restressed that it is not the intent of this work to determine the Johnson-Cook, Zerilli-Armstrong, or Steinberg-Guinan constants for OFE copper, but rather to present an instrumented anvil-on-rod impact experimental method of validating these models and similar ones against the entire deformation process. The development of such path-linked models is a critical step in understanding the behavior of even more complex material systems, whereby dynamic mechanical testing may be complicated by fracture or other physical/chemical changes.

\section{CONCLUSIONS}

A method of model validation using a combination of high-speed camera images of transient deformation states, velocity interferometry data, and finite-element simulations has been presented for the instrumented Taylor anvil-on-rod impact test performed at 83 and $205 \mathrm{~m} / \mathrm{s}$. Matching the specimen rear free surface velocity through adjustment of several parameters for the Johnson-Cook, Zerilli-Armstrong, and Steinberg-Guinan constitutive models resulted in accurate prediction of the final deformed state of the impacted samples at $83 \mathrm{~m} / \mathrm{s}$, as well as the transient specimen lengths, though examination of the crushed-end transient profiles revealed a decent fit accomplished only by the Johnson-Cook model. For the higher velocity $(205 \mathrm{~m} / \mathrm{s})$ impact experiment, the ZA model and the SG model reproduce the entire specimen profile to within $5 \%$ throughout the deformation event, while the JC model generates considerably large errors. In consideration of the respective constitutive models, the Johnson-Cook model is not path linked as is. Adjusting the parameters does not make it applicable to higher velocities, and correspondingly, higher strain rates. The ZerilliArmstrong model does not match the transient profiles at $83 \mathrm{~m} / \mathrm{s}$, but matches both the transient and final profiles at the higher velocity. The Steinberg-Guinan model performs satisfactorily at low velocity, and very well at high velocity.

The results illustrate that the instrumented anvil-on-rod impact tests employing the combined use of a high-speed camera to generate transient deformation states and velocity interferometry to monitor elastic-plastic wave propagation is necessary to ensure proper validation of path-linked constitutive models. 
IG. R. Johnson and W. H. Cook, Proceedings of the Seventh International Symposium on Ballistics, 1983, The Netherlands (Am. Def. Prep. Assoc. (ADPA)), pp. 541-547.

${ }^{2}$ F. J. Zerilli and R. W. Armstrong, J. Appl. Phys. 61, 1816 (1987).

${ }^{3}$ P. S. Follansbee and U. F. Kocks, Acta Metall. 36, 82 (1988).

${ }^{4}$ D. J. Steinberg, S. G. Cochran, and M. W. Guinan, J. Appl. Phys. 51, 1498 (1980).

${ }^{5}$ G. Taylor, Proc. R. Soc. London, Ser. A 194, 289 (1948).

${ }^{6}$ J. B. Hawkyard, Int. J. Mech. Sci. 11, 313 (1969).

${ }^{7}$ M. L. Wilkins and M. W. Guinan, J. Appl. Phys. 44, 1200 (1973).

${ }^{8}$ W. H. Gust, J. Appl. Phys. 53, 3566 (1982).
${ }^{9}$ L. M. Barker and R. E. Hollenbach, J. Appl. Phys. 43, 4669 (1972).

${ }^{10}$ I. Rohr, H. Nahme, and K. Thoma, J. Phys. IV 110, 513 (2003).

${ }^{11}$ I. Rohr, H. Nahme, and K. Thoma, Int. J. Impact Eng. 31, 401 (2005).

${ }^{12}$ B. D. Goldthorpe, A. L. Butler, and P. Church, J. Phys. IV 4, 471 (1994).

${ }^{13}$ P. J. Gould and B. D. Goldthorpe, J. Phys. IV 10, 39 (2000).

${ }^{14}$ S. Frechard, A. Lichtenberger, F. Rondot, N. Faderl, A. Redjaimia, and M. Adoum, J. Phys. IV 110, 9 (2003).

${ }^{15}$ S. M. Walley, P. D. Church, R. Townsley, and J. E. Field, J. Phys. IV 10, 69 (2000).

${ }^{16}$ Autodyn 2D, Version 6.0, Century Dynamics.

${ }^{17}$ H. Conrad, High-Strength Materials (Wiley, New York, 1965), pp. 884. 